\title{
Fathers' Childhood Experiences, Adult Mental Health Problems and Perceptions of Interactions With Their 36 Month-Old Children
}

\author{
Roni Mermelshtine ${ }^{1}$ \& Jacqueline Barnes ${ }^{1,2}$ \\ ${ }^{1}$ Department of Psychological Sciences, Birkbeck, University of London, UK \\ ${ }^{2}$ Tavistock and Portman NHS Trust, London, UK \\ Correspondence: Professor Jacqueline Barnes, Department of Psychological Sciences, Birkbeck, Malet Street, \\ London WC1E 7HX, UK. Tel: 44-207-079-0837. E-mail: jacqueline.barnes@bbk.ac.uk
}

\author{
Received: May 18, 2018 Accepted: June 5, 2018 Online Published: June 11, 2018 \\ doi:10.5539/jedp.v8n2p41 URL: http://doi.org/10.5539/jedp.v8n2p41
}

\begin{abstract}
Perceptions of poor care in the family of origin can relate to adverse mental health and poor adaptation for mothers but there is less evidence about fathers. This study investigated the relevance of fathers' recollections of their own parents (Generation 1) for their (Generation 2) current mental health symptoms and for interactions with their 3 -year-old children (Generations $2 / 3$ ), in a community sample of 482 British fathers. Recollections of G1 maternal and paternal behaviour were associated in uncontrolled correlations with G2 paternal mental health, but taking family social class and maternal (G2) mental health into account they did not significantly predict G2 fathers' mental health symptoms at 36 months postpartum, though a trend remained for G1 paternal care. Significant predictors were paternal depression symptoms in the first year postpartum and G2 mothers' current mental health. Predictors of more dysfunctional father-child (G2/G3) interactions at 36 months postpartum were working class status, recall of more G1 maternal controlling behaviour and more concurrent paternal mental health symptoms; predictors of less G2/G3 dysfunction were G2 paternal use of more positive discipline. Potential implications of the results for parenting support and advice are discussed, recognising that intergenerational transmission of parent-child relationships is likely for fathers.
\end{abstract}

Keywords: fathers, grandparents, mental health, parenting

\section{Introduction}

Research within social learning or attachment frameworks has tended to focus predominantly on single parent-child dyad behaviour (Ramchandani \& Iles, 2014) typically studying maternal behaviour, which may exaggerate the effects maternal caregivers have on child outcomes (Belsky, 1997). Literature focused on paternal contribution to child development has increased substantially (Lamb, 2012; Ramchandani \& Iles, 2014), but research addressing the intergenerational transmission of fathers' parenting practices remains less developed. This study focuses on the unique intergenerational transmission of the parenting of first generation (G1) mothers and fathers on present-day fathers' (G2) mental health and on their relationships with their preschool children (G3).

\subsection{Intergenerational Transmission of Parenting}

Intergenerational transmission of parenting refers to observed similarities in childrearing across generations (Serbin \& Karp, 2004), often explained with attachment or social learning theories (Belsky, Conger \& Capaldi, 2009). The former theory purports that the quality of caregiver-child relationships in family of origin determine present-day parents' child-rearing practices (Beaver \& Belsky, 2012; van IJzendoorn, 1992). The latter maintains that through observations and imitation of their G1 parents, G2 children come to practice the parenting styles they experienced in their family of origin (Capaldi, Pears, Patterson \& Owen, 2003). Such inter-generational similarities have been more commonly observed in negative parenting practices (Belsky et al., 2009; Capaldi et al., 2003; Serbin \& Karp, 2004), but positive child rearing practices are also likely to be transmitted across generations (Belsky Jaffe, Sligo, Woodward \& Silva, 2005; Chen \& Kaplan, 2001; Kerr, Capaldi, Pears \& Owen., 2009; Madden et al., 2015; Neppl, Conger, Scaramella \& Ontai, 2009). In one prospective US study, focused primarily on young disadvantaged G2 fathers with high levels of antisocial behaviour, G1 parents' discipline and monitoring were shown to be associated with G2 fathers' parenting practices; G1 poor care and harsh discipline was associated with similar patterns of behaviour in G2 fathers' behaviours (Capaldi et al., 2003; Capaldi, Pears, Kerr \& Owen, 
2008), and G1 constructive parenting with G2 more positive fathering (Kerr, et al., 2009). However unique relevance of G1 mother and father separately was not examined.

Recent research has investigated the separate transmission of G1 mothers' and fathers' parenting practices to G2 mothers and fathers finding that there was transmission only of first-generation mothers' parenting, not fathers' (Madden et al., 2015). Using the Parental Bonding Instrument to describe G1 parenting (PBI; Parker, Tupling \& Brown, 1979) Madden and her colleagues found that G1 paternal caregiving was not associated with G2 parenting for either mothers or fathers, yet G1 maternal affection and control were predictive of G2 fathers' positive parenting behaviours, and G2 mothers' and fathers' negative behaviours. These findings suggest that G2 parents, regardless of their gender, may emulate the care received in family of origin from their mothers, but not from fathers. In the context of attachment theory, the effects of paternal attachment representations on child attachment security may be less profound than those of maternal representations (van IJzendoorn, 1992). Some, in fact, have argued that father' attachments indirectly predict children's attachment security through their effects on maternal representations (van IJzendoorn \& Wolff, 1997).

Although first-generation fathers' care is found to be less meaningful for predicting G2 parent behaviours, evidence suggests that the practices of second-generation (current) fathers is to some extent a function of the parenting experienced in family of origin. A qualitative study of men who were the primary caregivers of their infants found that many of the reasons for taking that role were related to their own father, either to emulate his good practice or to avoid his (usually distant) style of parenting (West et al., 2009).

\subsection{The Role of G1 Fathering in G2 Parents'Subsequent Psychological Wellbeing}

The unique relationship between paternal care experienced by men and their subsequent psychological wellbeing is not entirely clear, though it is suggested that fathers' early involvement has a significant role in protecting from subsequent maladjustment (Flouri \& Buchanan, 2003). Indeed, some have shown that adult men who reported more positive relationships with their parents were likely to present fewer psychological problems, whilst the father-son relationship was the only significant factor associated with sons' psychological distress (Barnett, Marshall \& Pleck, 1992).

Risk experienced by fathers may intensify the effects of intergenerational influences while positive adjustment in adulthood may moderate the detrimental effects of early risk. In a sample of disadvantaged G2 mothers, Travis and Combs-Orme (2007) found that those who experienced poor parental bonds in their family of origin, but presented adaptive psychological functioning in adulthood, did not differ from G2 mothers who reported positive care and positive functioning. Travis and Combs-Orme labelled these mothers 'resilient', since they were similar to the low-risk group in their interactions with their G3 children, their parenting-related stress and maladaptive behaviour. Even though fathers' attachment representations are said to show very similar pattern to those of mothers (Van IJzendoorn \& Bakermans-Kranenburg, 1996) similar research with fathers is yet to be carried out.

The findings by Travis and Combs-Orme (2007) are of interest as they imply that the risk associated with poor parental bonding, such as depression (Murphy, Wickramaratne \& Weissman, 2010), can be overcome. Poor care experiences may promote positive adaptation in some, but for others early adversity will increase vulnerability to psychopathology (Beaver \& Belsky, 2012; Daskalakis, Bagot, Parker, Vinkers, \& de Kloet, 2013). For example, Parker and colleagues (1979) found that children who experienced higher levels of depression reported their parents as having been more controlling (intrusive) and less caring (rejecting); such bonding type is said to have a unique contribution to depression and is least conducive to positive development. It is possible that, for men in particular, paternal rejection in family of origin predicts elevated levels of depressive symptomatology, whilst G1 maternal negative care is more likely to be associated with G2 fathers rejecting behaviours (Whitbeck et al., 1992). If their mental health problems persist, and have links with their childhood experiences, their current mental health may mediate any potential relevance of G1 parents' behaviour for their own parent-child interactions.

\subsection{Relevance of Paternal Mental Health Symptoms for Child Development}

Paternal care has been associated with children's socio-emotional development (Flouri \& Buchanan, 2003; Flouri \& Malmberg, 2011; Ramchandani, van IJendoorn \& Bakermans-Kranenburg, 2010). This relationship is often understood in the context of fathers' psychological wellbeing, with children whose fathers show elevated levels of psychopathology at greater risk of maladjustment (Narayanan \& Nærde, 2016; Nath, Russell, Ford, Kuyken \& Psychogiou, 2015; Ramchamdani, et al., 2008; Ramchandani et al., 2010; Smith, Eryigit-Madzwamuse \& Barnes, 2013). It has been shown that men who present more depressive symptoms report higher levels of dysfunction in their relationship with their child (Kane \& Garber, 2004; Nath et al., 2015).

Much is known of the detrimental effects of maternal depression on parenting practices (Sohr-Preston \& 
Scaramella, 2006; Wilson \& Durbin, 2010) and children's behavioural problems (Goodman, Rouse, Connell, Broth, Hall \& Hayward, 2011). However, the role of paternal mental health remains less well understood (Herring \& Kalsow, 2002; Weitzman, Rosenthal \& Liu, 2011). Recent findings suggest that fathers' depressive symptoms, similar to those exhibited by mothers, are uniquely implicated in child problem behaviour, through their impact on their parenting behaviour (Edmondson et al., 2010; Ramchandani et al., 2005; Smith et al., 2013; Ramchandani \& Psychgiou, 2009; Weitzman et al., 2011; Wilson \& Durbin, 2010).

Although the prevalence of depressive symptoms in men is lower than that for women, men still experience significant levels of depression across their lifetime, especially throughout the child-rearing years (Kane \& Garber, 2004). Fathers in the pre- and postpartum periods experience similar hormonal changes to those experienced by mothers, such as decrease in testosterone and increase in prolactin and cortisol (Storey, Walsh, Quinton \& Wynne-Edwards, 2000). Neuroimaging research has shown that fathers demonstrate the same increase in the volume of grey matter in three specific areas of the brain as mothers and that this increase is associated with fewer depressive symptoms postpartum (Swain, Dayton, Kim, Tolman \& Volling, 2014). Goodman and Gotlib's (1999) model of transmission of risk considered fathers' psychological functioning for its moderating effects, not as a factor directly affecting child outcomes, an assertion similar to that of van IJzendoorn and Wolff's (1997) conclusion regarding the indirect effects of fathers' attachment representations.

It is also likely that fathers' mental health and their relationship with children is detrimentally affected by mothers' mental health and the quality of the couple relationship (Smith et al., 2013). This could be understood in the context of dynamic developmental systems theory (Capaldi et al., 2008), suggesting that the psychological wellbeing of the romantic partner (the child's mother) may significantly influence father care behaviours towards his child in contributing to elevated levels of risk.

\subsection{Aims of the Study}

In summary, grandparental (G1) parent behaviour may or may not directly relate to G2 fathers' parenting strategies, but it may predict second-generation paternal psychological wellbeing, likely to influence G2 parenting. Given that parents' psychological state can be linked to parenting behaviour and to child negative developmental outcomes for both mothers (Sohr-Preston \& Scaramella, 2006) and fathers (Nath et al., 2015; Ramchandani \& Psychgiou, 2009; Smith et al., 2013; Weitzman et al., 2011; Wilson \& Durbin, 2010), it is important to understand the associations between G1 parental care and G2 fathers' psychological states, and between their own care, current psychological states and their parenting, looking at the relevance of each G1 parent separately.

The present study tested the unique contribution of G1 fathers' and mothers' control and affection measured by the Parental Bonding Instrument (Parker, Tupling \& Brown, 1979) to (1) G2 fathers' psychological wellbeing and (2) G2 fathers' dysfunctional interactions with their G3 preschool child, taking their psychological state and that of their partner into account. The following hypotheses were tested:

1) Retrospective reports of G1 maternal and paternal affection and control will predict G2 fathers' reported psychological state at 36 months postpartum, taking earlier depression symptoms of $\mathrm{G} 2$ mother and father into account.

2) Retrospective reports of G1 maternal and paternal affection and control will predict G2 fathers' reported dysfunctional interaction with their (G3) preschool child at 36 months postpartum, taking G2 fathers' and mothers' current mental health symptoms and G2 fathers' methods of discipline into account.

\section{Methods}

\subsection{Participants and Procedure}

The FCCC is a longitudinal study of the possible effects of childcare on development in a community sample of 1201 children and their families. The Royal Free and University College Medical School and Oxford University gave ethical approval. Mothers were recruited in antenatal clinics in North London and Oxfordshire. Eligibility criteria were: mothers aged 16 or over and infant full term singleton with no congenital abnormalities (see Malmberg et al., 2005). All participants provided written informed consent. Data were collected following the child's birth at 3, 10, 18, 30, 36 and 51 months. During home visits maternal interviews and questionnaires were administered. Paternal questionnaires were left at home visits and returned by post, with two reminders sent if they were not received. The sample sizes for mothers at follow-up were 1077 (89.7\%), $1050(87.4 \%), 1036(86.3 \%)$, and $1040(86.6 \%)$ at $10,18,36$ and 51 months respectively. Of the 1,201 participants, 1,085 were families with a father in the home, either married $(\mathrm{N}=814)$ or cohabiting $(\mathrm{N}=271)$ and of those $713(65.7 \%)$ fathers completed questionnaires at 3 months, $738(68.0 \%)$ at 10 months, $617(56.9 \%)$ at 18 months, $637(58.7 \%)$ at 36 months and $714(65.8 \%)$ at 51 months. This study sample included 482 families with father questionnaires returned at 3, 10, 18 
and 36 months. The majority of parents were of white ethnic background (80\%) and $61 \%$ of families had at least one parent educated to a degree level or above (Malmberg et al., 2005).

\subsection{Measures}

\subsubsection{Demographic Characteristics}

Information was collected at 3 months postpartum on child gender and family occupational status (the highest of mother and father), defined according to the Computer Assisted System for Occupational Coding (CASOC; Rose \& O'Reilly, 1998) into three classes: working class or long-term unemployed; intermediate; professional or managerial.

\subsubsection{G2 Parents' Psychological State}

G2 parents' psychological state was assessed at three time points. At 3 and 10 months postpartum mothers and fathers completed the 10 item Edinburgh Postnatal Depression Scale (Cox, Holden and Sagovsky, 1987) asking about symptoms in the previous 7 days with 4-point response scales (0-1-2-3) giving a range of possible scores from 0 to 30 and a cut-off point of 13 to determine marked depressive symptomatology (Matthey, 2008). The EPDS had been validated in large community samples with mothers (Cox, Chapman, Murray \& Jones, 1996; Murray \& Carothers, 1990) and more recently as a screening tool with male participants (Edmondson et al., 2010; Matthey, Barnett, Kavanagh, \& Howie, 2001). Internal consistencies were $\alpha=.84$ and $\alpha=.80$ at 3 months and $\alpha$ $=.86$ and $\alpha=.83$ at 10 months, for mothers and fathers respectively. A mean EPDS score across both time-points in the first year was calculated.

At 36 months postpartum parents completed the 12-item General Health Questionnaire (GHQ; Goldberg \& Williams, 1988), reporting on non-psychotic mental health symptoms over the previous few weeks, using a 4-point (0-3) Likert scale (less than usual, no more than usual, rather more than usual, or much more than usual). Scores can range between 0-36, with higher scores representing more symptoms. Internal consistencies were $\alpha=.85$ and $\alpha$ $=.86$ respectively for the mother and father 36 month GHQ.

\subsubsection{G2 Parents' Reports on G1 Parental Bonding}

At 18 months postpartum, fathers completed the 25-item Parental Bonding Instrument (PBI; Parker, Tupling \& Brown, 1979) reporting on their recall of G1 maternal and paternal affection and control experienced up to the age of 16. The PBI items have a 4-point scale asking how closely each statement describes G1 mother and father behaviour. Affection (12-items) covers closeness, warmth, empathy and reciprocity, higher scores representing more positive parenting; Control (13-items) covers intrusiveness and infantilisation, higher scores representing adverse, controlling parenting. Internal consistency ranged between $\alpha=.88$ and $\alpha=.99$.

\subsubsection{G2 Paternal Discipline}

Mothers reported on the style of discipline used by G2 fathers at 36 months. Using questions developed for the study they were asked (yes $=1 /$ no $=0$ ) whether each of seven discipline strategies was used by the father when the child was uncooperative or difficult, covering four negative strategies: verbal reprimand, threats, loss of privilege, time out; and three positive: jokes/humour, distraction, reasoning.

\subsubsection{G2/G3 Parent-Child Quality of Interaction}

At 36 months postpartum fathers completed the 12-item 'Parent-child dysfunctional interaction' scale from the Parenting Stress Index- Short Form (PSI-SF; Abidin, 1997). Statements with a 5 point (strongly agree to strongly disagree) response scale describe feelings such as "sometimes I feel that my child doesn't like me", "my child is not able to do as much as I expected" and "sometimes my child does things that bother me just to be mean" (range 12-60; internal consistency $\alpha=.87$ ).

\subsection{Analytic Strategy}

Using SPSS 22, the initial step was to compare the demographic characteristics of the study sample with complete information from fathers at 3,10, 18 and 36 months postpartum $(\mathrm{N}=482)$ and the remainder of the FCCC sample $(\mathrm{N}=719)$. The extent to which both parents reported symptoms of depression above the cut-off for the EPDS at the start of the study (3 months) were also compared. Then bivariate Pearson correlations were conducted between fathers' recalled experiences of care in their family of origin (G1 parenting), fathers' and mothers' (G2) psychological state, G2 fathers' discipline and G2 fathers' reported dysfunctional interactions with their (G3) 36 month-old children.

Stepped multiple regressions were conducted including all those factors found to be significantly associated with the outcomes in uncontrolled analyses, first to identify significant predictors of paternal mental health symptoms at 
36 months postpartum, then to identify predictors of father-child dysfunctional interactions at 36 months postpartum. In each case the final stage of the regression was to simultaneously enter predictors identified as significant in the stepped analysis.

\section{Results}

\subsection{Participant Characteristics}

A comparison between the 482 families with complete father questionnaire information from 3 to 36 months and the remainder of the sample $(\mathrm{N}=719)$ found that the study families differed significantly on demographic characteristics (see Table 1). Both parents were likely to be older (fathers: $t=3.27, p<.05$; mothers: $t=6.04, p<.01$ ); fathers and mothers were more likely to be of white ethnic background (fathers: $x^{2}(1)=63.07, p<.01$; mothers: $\left.x^{2}(1)=50.46, p<.01\right)$; parents were more likely to be living together at 3 months $\left(x^{2}(1)=75.34, p<.01\right)$; and the family socio-economic status (highest of father or mother) was more likely to be professional or managerial $\left(x^{2}(2)\right.$ $=81.42, p<.001)$. Child gender did not differentiate the two groups.

Some differences were also identified in the proportion of parents with marked symptoms of depression (EPDS score $>13$ ) in the first year postpartum (see Table 1). At 3 months postpartum fewer mothers (but not fathers) in the study sample were above the EPDS cut-off $\left(x^{2}(1)=6.38, \mathrm{p}<.05\right)$ compared to families without full father questionnaire data; at 10 months fewer mothers and fathers were above the EPDS cut-off (mothers $x^{2}(1)=8.67$, $\mathrm{p}<.01$; fathers $\left.x^{2}(1)=8.40, \mathrm{p}<.01\right)$. Thus, for subsequent analyses, given the relatively small proportion above the cut-off, the continuous scores EPDS scores were used rather than categorical status.

Table 1. Comparison between families with complete father questionnaire data at 3,10,18 and 36 months and the remainder of the Families, Children and Child Care (FCCC) sample ( $t$ test values for continuous variables and Chi Square for categorical variables)

\begin{tabular}{llll}
\hline & Completed & Remainder of FCCC & $\mathrm{t} / x^{2}$ \\
\cline { 2 - 4 } & $\mathrm{N}=482$ & $\mathrm{~N}=719$ & \\
\hline Child gender & & & \\
Girl (\%) & $240(49.8)$ & $362(50.3)$ & \\
Boy (\%) & $242(50.2)$ & $357(49.7)$ & $75.34^{* *}$ \\
\hline Parents live together at 3 months & & & \\
Yes (\%) & $479(99.4)$ & $606(84.3)$ & \\
No (\%) & $3(.06)$ & $113(15.7)$ & $63.07 * *$ \\
\hline Minority status & & & $50.46^{* *}$ \\
Father & & & \\
Yes (\%) & $55(11.4)$ & $224(31.2)$ & $3.27 * *$ \\
No (\%) & $427(88.6)$ & $495(68.8)$ & $6.04 * *$ \\
\hline Mother & & & \\
Yes (\%) & $52(10.8)$ & $200(27.8)$ & \\
No (\%) & $430(89.2)$ & $519(72.2)$ & \\
\hline Mean parental age at birth & & & \\
Father (SD) & $34.7(5.9)$ & $33.5(6.1)$ & \\
Mother (SD) & $32.1(4.5)$ & $30.3(5.6)$ & \\
\hline Family SES & & & \\
Working class (\%) & $57(22.7)$ & $216(30)$ & $151(21)$ \\
Intermediate (\%) & $67(13.9)$ & $352(49)$ & \\
Professional/managerial (\%) & $358(74.3)$ & & \\
\hline
\end{tabular}




\section{Parental depression symptoms}

Mother mean EPDS 3-10 months total (SD)

Father mean EPDS 3-10 months total (SD)

Mother EPDS above cut-off at 3 months (\%)

Mother EPDS above cut-off at 10 months (\%)

Father EPDS above cut-off at 3 months (\%)

Father EPDS above cut-off at 10 months (\%)

$\begin{array}{lll}6.1(3.7) & 7.1(4.1) & 4.57^{* *} \\ 5.3(3.4) & 5.7(3.9) & n . s . \\ 36(7.5) & 86(12) & 6.38^{*} \\ 37(7.9) & 69(13.9) & 8.67^{* *} \\ 18(4.6) & 15(5.2) & n . s . \\ 19(4.3) & 28(9.6) & 8.40^{* *}\end{array}$

SD - Standard Deviation, EPDS - Edinburgh Postnatal Depression Scale, $x^{2}$ Chi Square, n.s. not significant, ${ }^{*} \mathrm{p}<.05, * *$ $\mathrm{p}<.01$

\subsection{Uncontrolled Associations Between Covariates and Outcomes}

Bivariate correlations confirmed that, if fathers reported more G1 parental affection from either their mother or father, then they were likely, subsequent to their own child's birth, to have fewer depression symptoms in the first year (mother care $\mathrm{r}=-.16$, father care $\mathrm{r}=-.17$ ) and fewer mental health symptoms at 36 months postpartum (mother care $\mathrm{r}=-.14$; father care $\mathrm{r}=-.16$ ) (see Table 2). In contrast recalling more G1 parental control from either parent, G2 fathers were likely to have more mental health symptoms at both time points (first year: mother control $\mathrm{r}=.16$, father control $\mathrm{r}=.12 ; 36$ months postpartum: mother control $\mathrm{r}=.11$, father control $\mathrm{r}=.11)$. Maternal $(\mathrm{G} 2)$ mental health symptoms were significantly associated with concurrent paternal (G2) symptoms in the first year (r $=.29)$ and at 36 months postpartum $(\mathrm{r}=.21)$. Neither positive nor negative discipline used by fathers at 36 months was associated with their mental health symptoms at 36 months.

More control by each G1 parent was positively associated with G2/G3 dysfunctional father-child interaction at 36 months (mother control $\mathrm{r}=.17$; father control $\mathrm{r}=.13$ ) whereas more G1 care by either parent was negatively associated with $\mathrm{G} 2 / \mathrm{G} 3$ dysfunctional interaction (mother care $\mathrm{r}=-.19$; father care $\mathrm{r}=-.11$ ) (see Table 2). There was no significant association with G2 maternal mental health at either time point and G2/G3 father-child dysfunctional interaction at 36 months but paternal mental health was associated with father-child dysfunctional interaction at 36 months (depression symptoms first year $r=.15$, mental health symptoms 36 months $r=.17$ ). Paternal (G2) use of positive discipline at 36 months was negatively associated with G2/G3 dysfunctional father-child interaction $(r=-.13)$ but use of negative discipline was not related $(r=0.01$; see Table 2$)$.

Table 2. Uncontrolled associations between study covariates, paternal mental health symptoms and father-child dysfunctional interactions at 36 months postpartum

\begin{tabular}{|c|c|c|c|c|c|c|c|c|c|c|c|c|}
\hline & 1 & 2 & 3 & 4 & 5 & 6 & 7 & 8 & 9 & 10 & 11 & 12 \\
\hline \multicolumn{13}{|l|}{ 1. G2 Family SES } \\
\hline 2. G1 Father care & .00 & & & & & & & & & & & \\
\hline 3. G1 Father control & -.06 & $-.43 * *$ & & & & & & & & & & \\
\hline 4. G1 Mother care & .08 & $.45^{* *}$ & $-.21 * *$ & & & & & & & & & \\
\hline 5. G1 Mother control & -.02 & $-.34 * *$ & $.53^{* *}$ & $-.38 * *$ & & & & & & & & \\
\hline $\begin{array}{l}\text { 6. G2 Father positive discipline } 36 \\
\text { months }\end{array}$ & $.19 * *$ & -.06 & .08 & .06 & .01 & & & & & & & \\
\hline $\begin{array}{l}\text { 7. G2 Father negative discipline } 36 \\
\text { months }\end{array}$ & .01 & .08 & $.12 * *$ & .03 & -.01 & $.10^{*}$ & & & & & & \\
\hline $\begin{array}{l}\text { 8. G2 Mother depressive symptoms } \\
\text { 3-10 months }\end{array}$ & -.08 & $-.17 * *$ & .06 & $-.11 *$ & .05 & .01 & $-.12 * *$ & & & & & \\
\hline $\begin{array}{l}\text { 9. G2 Mother mental health } \\
\text { symptoms } 36 \text { months }\end{array}$ & .08 & $-.11^{*}$ & .07 & -.02 & .03 & .02 & -.02 & $.37 * *$ & & & & \\
\hline $\begin{array}{l}\text { 10. G2 Father depressive symptoms } \\
3-10 \text { months }\end{array}$ & -.02 & $-.17 * *$ & $.12 *$ & $-.16^{* *}$ & $.16^{* *}$ & .05 & -.09 & $.29 * *$ & $.14 * *$ & & & \\
\hline $\begin{array}{l}\text { 11. G2 Father mental health } \\
\text { symptoms } 36 \text { months }\end{array}$ & $.10^{*}$ & $-.16^{* *}$ & $.11 *$ & $-.14 * *$ & $.11^{*}$ & -.03 & -.03 & $.17 * *$ & $.21 * *$ & $.37 * *$ & & \\
\hline
\end{tabular}


12. G2-G3 Father-child dysfunctional interaction 36 months

\begin{tabular}{lccccccccccccc}
\hline $\begin{array}{l}\text { 12. G2-G3 Father-child dysfunctional } \\
\text { interaction 36 months }\end{array}$ & $-.10^{*}$ & $-.11^{*}$ & $.13^{* *}$ & $-.19^{* *}$ & $.17^{* *}$ & $-.13^{* *}$ & -.01 & .05 & .06 & $.15^{* *}$ & $.17^{* *}$ & \\
\hline Mean & 2.62 & 22.90 & 9.56 & 27.21 & 11.42 & .67 & 1.41 & 6.07 & 10.43 & 5.32 & 10.18 & 1.43 \\
SD & .69 & 8.05 & 6.36 & 7.08 & 7.12 & .72 & 1.04 & 3.74 & 4.33 & 3.40 & 4.51 & .45 \\
\hline
\end{tabular}

Note. G1 Grandparent generation, G2 parent generation, G3 child generation, SES socioeconomic status; SD standard deviation, ${ }^{*} \mathrm{p}<.05, * * \mathrm{p}<.01$.

\subsection{Predictors Based on Multiple Regression Analyses}

After taking into account social class in the regression analysis (intermediate class less depression that professional class), G1 parenting by mother or father did not predict G2 paternal mental health symptoms at 36 months except that less G1 paternal care was marginally associated with more depressive symptoms (Standardized Beta $=-.10, p$ $=.055$; see Table 3). Taking into account G2 maternal symptoms (first year and 36 months, step 3) and paternal symptoms (first year, step 4), 18\% of the variance in G2 paternal mental health symptoms at 36 months postpartum was explained, with only two significant predictors remained: paternal depression in the child's first year (Standardized Beta $=.33$ ), and maternal mental health symptoms at 36 months (Standardized Beta $=.14$; see Table $3)$. Intermediate class and G1 father care remained as trends $(\mathrm{p}<.10)$.

Table 3. Results of multiple regression analyses to predict paternal mental health symptoms at 36 months postpartum

\begin{tabular}{|c|c|c|c|c|c|c|c|c|c|c|}
\hline \multirow{3}{*}{$\begin{array}{l}\mathrm{N}=444 \\
\text { Variable }\end{array}$} & \multicolumn{8}{|c|}{ Stepped multiple regressions } & \multirow{2}{*}{\multicolumn{2}{|c|}{$\begin{array}{l}\begin{array}{l}\text { Simultaneous } \\
\text { regression }\end{array} \\
\text { Model } 5\end{array}$}} \\
\hline & \multicolumn{2}{|c|}{ Model 1} & \multicolumn{2}{|c|}{ Model 2} & \multicolumn{2}{|c|}{ Model 3} & \multicolumn{2}{|c|}{ Model 4} & & \\
\hline & $\boldsymbol{\beta}$ & $95 \% \mathrm{CI}$ & $\beta$ & $95 \% \mathrm{CI}$ & $\boldsymbol{\beta}$ & $95 \% \mathrm{CI}$ & $\boldsymbol{\beta}$ & $95 \% \mathrm{CI}$ & $\boldsymbol{\beta}$ & $95 \% \mathrm{CI}$ \\
\hline \multicolumn{11}{|l|}{ Family demographics } \\
\hline Working class & -.07 & $(-.21, .04)$ & $-.08 \dagger$ & $(-.20, .02)$ & $-.09 \dagger$ & $(-.24, .01)$ & -.07 & $(-.21, .02)$ & -.07 & $(-.20, .03)$ \\
\hline Intermediate class & $-.11 *$ & $(-.23,-.02)$ & $-.10^{*}$ & $(-.20,-.00)$ & $-.08 \dagger$ & $(-.20, .01)$ & $-.08 \dagger$ & $(-.19, .00)$ & $-.08 \dagger$ & $(-.19, .00)$ \\
\hline \multicolumn{11}{|l|}{ G1 Parenting } \\
\hline Father care & & & $-.10 \dagger$ & $(-.20, .02)$ & -.06 & $(-.17, .05)$ & -.05 & $(-.15, .06)$ & $-.09 \dagger$ & $(-.17, .00)$ \\
\hline Father control & & & .04 & $(-.07, .16)$ & .04 & $(-.08, .16)$ & .03 & $(-.08, .14)$ & & \\
\hline Mother care & & & -.08 & $(-.19, .03)$ & -.09 & $(-.20, .02)$ & -.06 & $(-.16, .05)$ & & \\
\hline Mother control & & & .03 & $(-.09, .15)$ & .03 & $(-.09, .14)$ & .01 & $(-.10, .12)$ & & \\
\hline \multicolumn{11}{|l|}{$\begin{array}{l}\text { G2 Mother's mental } \\
\text { health }\end{array}$} \\
\hline $\begin{array}{l}\text { 3-10 months depression } \\
\text { symptoms }\end{array}$ & & & & & $.10 \dagger$ & $(-.00, .20)$ & .00 & $(-.10, .10)$ & .00 & $(-.10, .10)$ \\
\hline $\begin{array}{l}36 \text { months mental } \\
\text { health symptoms }\end{array}$ & & & & & $.15^{* *}$ & $(.05, .25)$ & $.14 * *$ & $(.05, .26)$ & $.14 * *$ & $(.05, .23)$ \\
\hline \multicolumn{11}{|l|}{$\begin{array}{l}\text { G2 Father's mental } \\
\text { health }\end{array}$} \\
\hline $\begin{array}{l}3-10 \text { months depression } \\
\text { symptoms }\end{array}$ & & & & & & & $.33^{* *}$ & $(.24, .42)$ & $.34 * *$ & $(.25, .43)$ \\
\hline $\mathrm{R}^{2}$ & $.015^{*}$ & & $.049 * *$ & & $.090 * *$ & & $.184 * *$ & & $181 * *$ & \\
\hline$\Delta \mathrm{R}^{2}$ Change & & & $.034 * *$ & & $.041 * *$ & & $.094 * *$ & & & \\
\hline Model F & $(2,44$ & 1) $=3.26$ & $(6,437$ & $=3.73$ & $(8,435$ & $=5.37$ & $(9,434)$ & $=10.89$ & $(6,437)$ & $=16.05$ \\
\hline
\end{tabular}

Note. G1 Grandparent generation, G2 parent generation, G3 child generation, SES socioeconomic status; Simultaneous model includes only variable significant at the $\mathrm{p}<.10$ in one of the four stepped regression models. $\dagger \mathrm{p}<.10,{ }^{*} \mathrm{p}<.05,{ }^{* *} \mathrm{p}<.01$. 
Less of the variance in father-child (G2/G3) dysfunctional interaction at 36 months postpartum was explained in the final model (9\%). It was more likely in working class families, if G2 fathers recalled more G1 maternal over-control, and if G2 fathers had more mental health symptoms at 36 months postpartum (see Table 4). It was less likely if the $\mathrm{G} 2$ fathers currently used more positive discipline strategies and to a lesser extent if they recalled more G1 maternal care (marginal $p=.073$ ) (see Table 4).

Table 4. Results of multiple regressions to predict father-child dysfunctional interaction at 36 months postpartum

\begin{tabular}{|c|c|c|c|c|c|c|c|c|c|c|}
\hline \multirow{3}{*}{$\begin{array}{l}\mathrm{N}=457 \\
\text { Variable }\end{array}$} & \multicolumn{8}{|c|}{ Stepped multiple regression } & \multirow{2}{*}{\multicolumn{2}{|c|}{$\begin{array}{l}\begin{array}{l}\text { Simultaneous } \\
\text { regression }\end{array} \\
\text { Model } 5\end{array}$}} \\
\hline & \multicolumn{2}{|c|}{ Model 1} & \multicolumn{2}{|c|}{ Model 2} & \multicolumn{2}{|c|}{ Model 3} & \multicolumn{2}{|c|}{ Model 4} & & \\
\hline & $\boldsymbol{\beta}$ & $95 \% \mathrm{CI}$ & $\boldsymbol{\beta}$ & $95 \% \mathrm{CI}$ & $\boldsymbol{\beta}$ & $95 \% \mathrm{CI}$ & $\boldsymbol{\beta}$ & $95 \% \mathrm{CI}$ & $\boldsymbol{\beta}$ & $95 \% \mathrm{CI}$ \\
\hline \multicolumn{11}{|l|}{ Family demographics } \\
\hline G3 Gender (Girl) & -.03 & $(-.12, .06)$ & -.03 & $(-.12, .06)$ & -.30 & $(-.12, .06)$ & -.04 & $(-.12, .05)$ & & \\
\hline Working class & $.12 *$ & $(.04, .27)$ & $.10^{*}$ & $(.01, .24)$ & $.08 \dagger$ & $(-.02, .22)$ & $.10^{*}$ & $(.00, .24)$ & $.09 *$ & $(.00, .23)$ \\
\hline Intermediate class & .02 & $(-.08, .12)$ & .02 & $(-.08, .13)$ & .01 & $(-.09, .12)$ & .02 & $(-.08, .13)$ & & \\
\hline \multicolumn{11}{|l|}{ G1 Parenting } \\
\hline Father care & & & .00 & $(-.11, .11)$ & -.00 & $(-.11, .10)$ & .01 & $(-.10, .11)$ & & \\
\hline Father control & & & .02 & $(-.09, .13)$ & .03 & $(-.08, .15)$ & .02 & $(-.09, .13)$ & & \\
\hline Mother care & & & $-.11 *$ & $(-.22,-.01)$ & $-.11 \dagger$ & $(-.21, .00)$ & $-.09 \dagger$ & $(-.19, .02)$ & $-.09 \dagger$ & $(-.18, .01)$ \\
\hline Mother control & & & $.12^{*}$ & $(.00, .23)$ & $.11^{*}$ & $(.00, .22)$ & $.11 *$ & $(.00, .22)$ & $.12 *$ & $(.02, .21)$ \\
\hline \multicolumn{11}{|l|}{ G2 Father's parenting } \\
\hline Positive discipline & & & & & $-.11 *$ & $(-.20,-.02)$ & $-.10 *$ & $(-.19,-.01)$ & $-.11 *$ & $(-.19,-.02)$ \\
\hline \multicolumn{11}{|l|}{ G2 Father's mental health } \\
\hline $\begin{array}{l}36 \text { months mental health } \\
\text { symptoms }\end{array}$ & & & & & & & $.15^{* *}$ & $(.06, .25)$ & $.15^{* *}$ & $(.06, .23)$ \\
\hline $\mathrm{R}^{2}$ & .015 & & $.055^{* *}$ & & $.067 * *$ & & $.088 * *$ & & $.086^{* *}$ & \\
\hline$\Delta \mathrm{R}^{2}$ Change & & & $.039 * *$ & & $.012 *$ & & $.021 * *$ & & & \\
\hline Model F & $(3,45$ & $3)=2.37$ & $(7,449$ & $=3.73$ & $(8,448$ & $=4.02$ & $(9,447$ & $=4.77$ & $(5,451$ & $=8.45$ \\
\hline
\end{tabular}

Note. G1 Grandparent generation, G2 parent generation, G3 child generation, SES socioeconomic status; Simultaneous model include only variable significant at the $\mathrm{p}<.10$ in one of the four stepped regression models. $\dagger^{\dagger}<.10,{ }^{*} \mathrm{p}<.05,{ }^{* *} \mathrm{p}<.01$.

\section{Discussion}

The present study explored the individual contributions of G1 mothers' and fathers' parenting for the mental health of G2 fathers, likely to be relevant to their interactions with their G3 children. It also explored the direct relevance of G1 mothers' and fathers' parenting for G2/G3 father child interactions.

The first hypothesis was partially supported. G2 fathers' psychological state at 36 months postpartum was associated in uncontrolled correlations with G1 mothers and fathers' affection and with their control, but in the regression analysis, taking into account G2 maternal symptoms and G2 paternal depression symptoms in the first year postpartum, only G1 paternal affection remained significant. These results are somewhat comparable to Whitebeck and associates' (1992) findings that G1 paternal rejection (but not G1 maternal rejection) directly influenced G2 paternal mental health. These findings can be considered in the context of Goodman and Gotlib's (1999) model of transmission of psychopathology. The detrimental effect of father's mental health problems indirectly affect child outcomes through its influence on maternal mental health. For G2 fathers the mental health of their partner was a highly significant predictor of their own mental health, as found by Matthey and colleagues (2000). To better understand this relationship in the context of intergenerational transmission of parenting, aspects of the couple relationship that are associated with increases in depressive symptoms (Matthey et al., 2000; Smith et al., 2013) could be included in future investigations.

The second hypothesis was partially supported, indicating the greater relevance of G1 maternal than paternal 
behaviour, and the importance of current paternal mental health for father-child interactions. In line with Madden and colleagues (2015), our findings suggest that more controlling G1 mothering, but not fathering and to a lesser extent less affectionate G1 mothering (but again not fathering) were directly associated with G2 fathers' dysfunctional interactions with their 3-year-olds. To a certain extent the findings are also in keeping with Chen and Kaplan's (2001) findings, which suggest that experiences of positive parenting directly predict more positive parenting practices (or less dysfunction) in present-day parents. The fact that maternal controlling behaviour was the stronger predictor of subsequently dysfunctional interactions corresponds more closely however to the previous work by Capaldi and colleagues $(2003 ; 2008)$, which highlighted the relevance of G1 harsh discipline for the parenting behaviour of their G2 sons, and the related difficult temperament of G3 infants. The findings contrast with the qualitative study of the reasons why fathers decide to take a highly active role in their child's caregiving, which focus more on their relationship with their father (West et al., 2008). However, taking a role is not necessarily the same as the subsequent interactions. Actual parenting appears to be relates to a greater extent to fathers' own mothers and to their own current mental health. Although specific paternal parenting practices are less likely to be transmitted across generations to fathers, the effects of less affectionate paternal care may have implications for the male-offspring's mental health once they become parents.

\subsection{Strengths and Limitations}

Our results also add to the fairly limited UK-based literature on the intergenerational transmission of parenting. A major strength of the study is its considerably large sample size, from a community study rather than one based on clinical characteristics, which makes the findings more generalisable. Compared to much of the previous research, which has concerned at-risk populations such as men who had conduct disorder as children and who became parents at a young age (Capaldi et al., 2003; 2008), this study is the reverse. Those who completed all of the required questionnaires were in intact families, were older than the remainder of the sample, were more likely to be in professional occupations and mothers were less likely to have reported depression postpartum. Thus this study is a very low risk sample and illustrates that intergenerational influences on parenting are not only relevant for vulnerable and more extreme experiences of parenting but for the majority of families. The results show that even in a relatively advantaged sample, less positive experiences of care can have an adverse effect on G2 fathers' mental health and on their reported interactions with G3 children.

There are some limitations however. The study was based on a convenience sample which led to a bias towards more educated and older parents, known to have fewer parenting problems on average (Barnes, Gardiner, Sutcliffe $\&$ Melhuish, 2014). In addition the results have limited applicability to families with ethnic minority backgrounds and to alternative family forms, both of which are found in the UK. Retention of mothers in the study was good but from the outset the questionnaires received from fathers represented only about two thirds of families with a father in the home. A study directly involving fathers would have been preferable but the budget did not allow for separate visits to the homes at times when fathers were present.

The lower participation and follow-up for fathers may be relevant to the findings. Adults who report more negative relationships with their parents as children are more likely to present higher levels of depression (Murphy, Wickramaratne \& Weissman, 2010; Parker et al., 1979). There were no differences between the respondents in this study and the remainder of the FCCC study participant's in terms of paternal mental health immediately postpartum (3 months) but by 10 months postpartum fathers reporting more depression were less likely to continue in the study and complete the PBI when it was administered at 18 months. It could not be done before the child was born as full inclusion in the study did not take place until 3 months postpartum. Therefore, this study may underestimate the relevance of G1 parenting since men will not have been included who might have been expected to report more negatively about their own parents. It is of note, however, that depressive symptoms were not found to bias the PBI's stability over a 20 -year period (Murphy et al., 2010) strengthening its validity as a psychometric instrument.

Another limitation is that the recollections of G1 parents were collected after the men had become parents. Research relying on retrospective reports is likely to be prone to memory distortion (Belsky et al., 2009) and could have been influenced by how well fathers thought they themselves were coping with parenthood. It could not be completed before the child was born as full inclusion in the study did not take place until 3 months postpartum. The PBI was administered in the second year postpartum to avoid the usual disruptions and stresses for parents with a young baby. Nevertheless, if families had been recruited in pregnancy that would have been a better time to ask the men to complete the PBI. The ideal study might start with the G1 generation so that their behaviour could be observed at the time, in particular the extent to which G1 fathers were actively involved in caregiving so that this could be linked to their own children as they become parents. Longitudinal study data could be sought to 
investigate this issue. However, one could argue, based on Fraiberg's conception of 'ghosts in the nursery' (Fraiberg, 1975; Fraiberg, Adelson, \& Shapiro, 1987) and on interventions taking that attachment approach (Acquarone, 1987; Cramer, 1998) that it is recall of what G1 parents were like that is most relevant to the next generation's parenting, not what G1 parents actually did.

\section{Conclusions}

The study showed that G2 fathers' mental health problems when their children were of preschool age were predicted to some extent by G1 fathers being a less affectionate caregiver but primarily by their own mental health in the year postpartum and by their partner's mental health concurrently. The present study also found intergenerational transmission effects of G1 mothers' affectionate care, reducing the likelihood of G2 fathers' dysfunctional interactions with their 3-year-olds. The results indicate two important ways that families could be supported to enhance parent-child interactions and consequently child development. First, reflecting other research focussing on paternal depression, this study has shown that difficult father-child relationships can be related to fathers' mental health problems. So clinicians, who may in general have more contact with mothers, would be sensible to enquire in detail about fathers' mental well-being. Second, some 'ghosts in the nursery' are likely to be present for fathers about their own mothers and interventions or therapies offered to families experiencing parenting difficulties would benefit from ensuring that fathers are asked about their own childhoods and in particular their relationship with their mother.

\section{Acknowledgements}

Data for this study were drawn from "The Families, Children and Childcare Study, funded by the Tedworth Charitable Trust and the Glass-House Trust and led by Penelope Leach and Jacqueline Barnes in London and Alan Stein, Kathy Sylva and Lars-Erik Malmberg in Oxford. www.familieschildrenchildcare.org

Data are freely available to the research community. For information contact Professor Jacqueline Barnes, Institute for the study of Children, Families \& Social Issues, Birkbeck, University of London.

\section{References}

Abidin, R. R. (1997). Parenting Stress Index: A measure of the parent-child system. In: C. P. Zalaquett \& R.J. Wood (Eds.), Evaluating stress: a book of resources (pp. 277-291). Lanham, MD, US: Scarecrow Press Inc.

Acquarone, S. (1987). Early interventions in disturbed mother-infant relationships. Journal of Mental Health, 8(4), 340-351. https://doi.org/10.1002/1097-0355(198724)8:4<340::AID-IMHJ2280080403>3.0.CO;2-0

Barnes, J., Gardiner, J., Sutcliffe, A. G., \& Melhuish, E. (2014). The parenting of young children by older mothers in the United Kingdom. European Journal of Developmental Psychology, 11(4), 397-419. https://doi.org/10.1080/17405629.2013.863728

Barnett, R. C., Marshall, N. L., \& Pleck, J. H. (1992). Men's multiple roles and their relationship to men's psychological distress. Journal of Marriage and the Family, 54(2), 358-367. https://doi.org/10.1177/019251392013004007

Beaver, K. M., \& Belsky, J. (2012). Gene-environment interaction and the intergenerational transmission of parenting: testing the differential-susceptibility hypothesis. Psychiatric Quarterly, 83(1), 29-40. https://doi.org/10.1007/s11126-011-9180-4

Belsky, J. (1997). Theory testing, effect - size evaluation, and differential susceptibility to rearing influence: The case of mothering and attachment. Child Development, 68(4), 598-600. https://doi.org/ 10.2307/1132110

Belsky, J., Conger, R., \& Capaldi, D. M. (2009). The intergenerational transmission of parenting: introduction to the special section. Developmental Psychology, 45(5), 1201-1204. doi.org/10.1037/a0016245

Belsky, J., Jaffee, S. R., Sligo, J., Woodward, L., \& Silva, P. A. (2005). Intergenerational transmission of warm sensitive - stimulating parenting: A prospective study of mothers and fathers of 3 - Year - Olds. Child Development, 76(2), 384-396. https://doi.org/ 10.1111/j.1467-8624.2005.00852.x

Capaldi, D. M., Pears, K. C., Kerr, D. C., \& Owen, L. D. (2008). Intergenerational and partner influences on fathers' negative discipline. Journal of Abnormal Child Psychology, 36(3), 347-358. https://doi.org/10.1007/s10802-007-9182-8

Capaldi, D. M., Pears, K. C., Patterson, G. R., \& Owen, L. D. (2003). Continuity of parenting practices across generations in an at-risk sample: A prospective comparison of direct and mediated associations. Journal of Abnormal Child Psychology, 31(2), 127-142. https://doi.org/10.1023/A:1022518123387 
Chen, Z. Y., \& Kaplan, H. B. (2001). Intergenerational transmission of constructive parenting. Journal of Marriage and Family, 63(1), 17-31. https://doi.org/10.1111/j.1741-3737.2001.00017.x

Cox, J. L., Chapman, G., Murray, D., \& Jones, P. (1996). Validation of the Edinburgh Postnatal Depression Scale (EPDS) in non-postnatal women. Journal of Affective Disorders, 39(3), 185-189. https://doi.org/10.1016/0165-0327(96)00008-0

Cox, J. L., Holden, J. M., \& Sagovsky, R. (1987). Detection of postnatal depression. Development of the 10-item Edinburgh Postnatal Depression Scale. The British Journal of Psychiatry, 150(6), 782-786. https://doi.org/10.1192/bjp.150.6.782

Cramer, B. (1998). Mother-infant psychotherapies: a widening scope in technique. Infant Mental Health Journal, 19, 151-167. https://doi.org/10.1002/(SICI)1097-0355(199822)19:2\%3C151::AID- IMHJ5\%3E3.0.CO;2-R

Daskalakis, N. P., Bagot, R. C., Parker, K. J., Vinkers, C. H., \& De Kloet, E. R. (2013). The three-hit concept of vulnerability and resilience: toward understanding adaptation to early-life adversity outcome. Psychoneuroendocrinology, 38(9), 1858-1873. https://doi.org/10.1016/j.psyneuen.2013.06.008.

Edmondson, O. J., Psychogiou, L., Vlachos, H., Netsi, E., \& Ramchandani, P. G. (2010). Depression in fathers in the postnatal period: Assessment of the Edinburgh Postnatal Depression Scale as a screening measure. Journal of Affective Disorders, 125(1), 365-368. https://doi.org/10.1016/j.jad.2010.01.069

Flouri, E., \& Buchanan, A. (2003). The role of father involvement in children's later mental health. Journal of Adolescence, 26(1), 63-78. https://doi.org/10.1016/S0140-1971(02)00116-1

Flouri, E., \& Malmberg, L. E. (2012). Fathers' involvement and preschool children's behavior in stable single-mother families. Children and Youth Services Review, 34(7), 1237-1242. https://doi.org/10.1016/j.childyouth.2012.02.020

Fraiberg, S., Adelson, E. \& Shapiro, V. (1975). Ghosts in the nursery. Journal of the American Academy of Child Psychiatry, 14 (3), 387-421. https://doi.org/10.1016/S0002-7138(09)61442-4

Fraiberg, S., Adelson, E., \& Shapiro, V. (1987). Ghosts in the nursery: a psychoanalytic approach to the problems of impaired infant-mother relationships. In S. Fraigerg, S. (Ed.), Selected writings of Selma Fraiberg (pp. 100-136). Columbus OH: Ohio State University Press.

Goldberg, D., \& Williams, P. (1988). A user's guide to the General Health Questionnaire. Windsor, UK: NFER-Nelson.

Goodman, S. H., \& Gotlib, I. H. (1999). Risk for psychopathology in the children of depressed mothers: a developmental model for understanding mechanisms of transmission. Psychological Review, 106(3), 458-490. doi.org/10.1037/0033-295X.106.3.458

Goodman, S. H., Rouse, M. H., Connell, A. M., Broth, M. R., Hall, C. M., \& Heyward, D. (2011). Maternal depression and child psychopathology: a meta-analytic review. Clinical Child and Family Psychology Review, 14(1), 1-27. https://doi.org/10.1007/s10567-010-0080-1.

Herring, M., \& Kaslow, N. J. (2002). Depression and Attachment in Families: A Child - Focused Perspective. Family Process, 41(3), 494-518. https://doi.org/10.1111/j.1545-5300.2002.41313.x

Kane, P., \& Garber, J. (2004). The relations among depression in fathers, children's psychopathology, and fatherchild conflict: A meta-analysis. Clinical Psychology Review, 24(3), 339-360. https://doi.org/10.1016/j.cpr.2004.03.004

Kerr, D. C., Capaldi, D. M., Pears, K. C., \& Owen, L. D. (2009). A prospective three generational study of fathers' constructive parenting: Influences from family of origin, adolescent adjustment, and offspring temperament. Developmental Psychology, 45(5), 1257. https://doi.org/10.1037/a0015863.

Lamb, M. E. (2012). Mothers, fathers, families, and circumstances: Factors affecting children's adjustment. Applied Developmental Science, 16(2), 98-111. https://doi.org/10.1080/10888691.2012.667344

Madden, V., Domoney, J., Aumayer, K., Sethna, V., Iles, J., Hubbard, I., ... \& Ramchandani, P. (2015). Intergenerational transmission of parenting: findings from a UK longitudinal study. The European Journal of Public Health, 25(3), 1-6. https://doi.org/10.1093/eurpub/ckv093

Malmberg, L.-E., Davies, B., Walker, J., Barnes, J., Sylva, K., Stein, A., \& Leach, P. (2005). The Families, Children and Child Care (FCCC) study in relation to area characteristics: Recruitment and sample description. Retrieved 01 June 2018 from http://www.familieschildrenchildcare.org/fccc_frames_home.html 
Matthey, S., Barnett, B., Kavanagh, D. J., \& Howie, P. (2001). Validation of the Edinburgh Postnatal Depression Scale for men, and comparison of item endorsement with their partners. Journal of Affective Disorders, 64(2), 175-184. https://doi.org/10.1016/S0165-0327(00)00236-6

Matthey, S., Barnett, B., Ungerer, J., \& Waters, B. (2001). Paternal and maternal depressed mood during the transition to parenthood. Journal of Affective Disorders, 60(2), 75-85. https://doi.org/10.1016/S0165-0327(99)00159-7

Murray, L., \& Carothers, A. D. (1990). The validation of the Edinburgh Post-natal Depression Scale on a community sample. The British Journal of Psychiatry, 157(2), 288-290. https://doi.org/10.1192/bjp.157.2.288

Murphy, E., Wickramaratne, P., \& Weissman, M. (2010). The stability of parental bonding reports: A 20-year follow-up. Journal of Affective Disorders, 125(1), 307-315. https://doi.org/10.1016/j.jad.2010.01.003

Nath, S., Russell, G., Ford, T., Kuyken, W., \& Psychogiou, L. (2015). Postnatal paternal depressive symptoms associated with fathers' subsequent parenting: findings from the Millennium Cohort Study. The British Journal of Psychiatry. https://doi.org/10.1192/bjp.bp.114.148379

Narayanan, M. K., \& Nærde, A. (2016). Associations between maternal and paternal depressive symptoms and early child behavior problems: testing a mutually adjusted prospective longitudinal model. Journal of Affective Disorders, 196, 181-189. https://doi.org/10.1016/j.jad.2016.02.020

Neppl, T. K., Conger, R. D., Scaramella, L. V., \& Ontai, L. L. (2009). Intergenerational continuity in parenting behaviour: Mediating pathways and child effects. Developmental Psychology, 45(5), 1241-1256. https://doi.org/10.1037/a0014850

Parker, G., Tupling, H., \& Brown, L. B. (1979). Parental bonding instrument (PBI). British Journal of Medical Psychology, 52, 1-10. https://doi.org/10.1111/j.2044-8341.1979.tb02487.x

Ramchandani, P., \& Iles, J. (2014). Commentary: Getting fathers into parenting programmes-a reflection on Panter - Brick et al.(2014). Journal of Child Psychology and Psychiatry, 55(11), 1213-1214. https://doi.org/10.1111/jcpp.12321

Ramchandani, P., \& Psychogiou, L. (2009). Paternal psychiatric disorders and children's psychosocial development. The Lancet, 374(9690), 646-653.

Ramchandani, P., Stein, A., Evans, J., \& O'Connor, T. G. (2005). Paternal depression in the postnatal period and child development: a prospective population study. The Lancet, 365(9478), 2201-2205. https://doi.org/10.1016/S0140-6736(09)60238-5

Ramchandani, P. G., van IJzendoorn, M. H., \& Bakermans-Kranenburg, M. J. (2010). Differential susceptibility to fathers' care and involvement: the moderating effect of infant reactivity. Family Science, 1(2), 93-101. https://doi.org/10.1080/19424621003599835

Rose, D., \& O'Reilly, K. (1998). Final report of the ESRC review of government social classifications. Swindon: Economic and Social Research Council and Office for National Statistics.

Serbin, L. A., \& Karp, J. (2004). The intergenerational transfer of psychosocial risk: Mediators of vulnerability and $\begin{array}{llllll}\text { resilience. Annual } & \text { Review }\end{array}$ https://doi.org/10.1146/annurev.psych.54.101601.145228

Smith, H. R., Eryigit - Madzwamuse, S., \& Barnes, J. (2013). Paternal Postnatal and Subsequent Mental Health Symptoms and Child Socio - Emotional and Behavioural Problems at School Entry. Infant and Child Development, 22(4), 335-348. https://doi.org/10.1002/icd.1800

Sohr-Preston, S. L., \& Scaramella, L. V. (2006). Implications of timing of maternal depressive symptoms for early cognitive and language development. Clinical Child and Family Psychology Review, 9(1), 65-83. https://doi.org/10.1007/s10567-006-0004-2

Storey, A. E., Walsh, C. J., Quinton, R. L., \& Wynne-Edwards, K. E. (2000). Hormonal correlates of paternal responsiveness in new and expectant fathers. Evolution and Human Behavior, 21(2), 79-95. doi.org/10.1016/S1090-5138(99)00042-2

Swain, J. E., Dayton, C. J., Kim, P., Tolman, R. M., \& Volling, B. L. (2014). Progress on the paternal brain: Theory, animal models, human brain research, and mental health implications. Infant Mental Health Journal, 35(5), 394-408. https://doi.org/10.1002/imhj.21471 
Travis W. J., \& Combs-Orme, T. (2007). Resilient parenting: Overcoming poor parental bonding. Social Work Research 31(3), 135-149. https://doi.org/10.1093/swr/31.3.135

van IJzendoorn, M. H. (1992). Intergenerational transmission of parenting: A review of studies in nonclinical populations. Developmental Review, 12(1), 76-99. https://doi.org/ 10.1016/0273-2297(92)90004-L

Weitzman, M., Rosenthal, D. G., \& Liu, Y. H. (2011). Paternal depressive symptoms and child behavioural or emotional problems in the United States. Pediatrics, 128(6), 1126-1134. https://doi.org/10.1542/peds.2010-3034

Whitbeck, L. B., Hoyt, D. R., Simons, R. L., Conger, R. D., Elder Jr, G. H., Lorenz, F. O., \& Huck, S. (1992). Intergenerational continuity of parental rejection and depressed affect. Journal of Personality and Social Psychology, 63(6), 1036-1045. https://doi.org/10.1037/0022-3514.63.6.1036

van IJzendoorn, M. H., \& Bakerman-Kranenburg M. J. (1996). Attachment representations in mothers, fathers, adolescents and clinical groups: A meta-analytic search for normative data. Journal of Consulting in Clinical Psychology, 64(1), 8-21. https://doi.org/ 10.1037//0022-006X.64.1.8

van IJzendoorn, M. H., \& Wolff, M. S. (1997). In search of the absent father-meta - analyses of infant - father attachment: a rejoinder to our discussants. Child Development, 68(4), 604-609. https://doi.org/10.2307/1132112

West, A., Lewis, S., Ram, B., Barnes, J., Leach, P., Sylva, K., Stein, A. and the FCCC team. (2008). Why do some fathers become primary caregivers for their infants? A qualitative study. Child: Care, Health and Development, 35(2), 208-216. https://doi.org/10.1111/j.1365-2214.2008.00926.x.

Wilson, S., \& Durbin, C. E. (2010). Effects of paternal depression on fathers' parenting behaviors: A meta-analytic review. Clinical Psychology Review, 30(2), 167-180. https://doi.org/10.1016/j.cpr.2009.10.007.

\section{Copyrights}

Copyright for this article is retained by the author(s), with first publication rights granted to the journal.

This is an open-access article distributed under the terms and conditions of the Creative Commons Attribution license (http://creativecommons.org/licenses/by/4.0/). 